\title{
Qualidade de vida de idosos atendidos em um centro de referência em Minas Gerais, Brasil
}

\section{Quality of life of elderly in a reference center in Minas Gerais, Brazil}

Juliana Souza Andrade ${ }^{1}$. Maria Fernanda Santos Figueiredo Brito². Luís Paulo Souza e Souza ${ }^{3}$. André Luiz Sena Guimarães ${ }^{4}$. Marcos Flávio Silveira Vasconcelos D’Angelo ${ }^{5}$. Carla Silvana de Oliveira e Silva ${ }^{6}$.

1 Enfermeira pela Universidade Estadual de Montes Claros (Unimontes), Montes Claros, Minas Gerais, Brasil. 2 Doutora em Ciências da Saúde, Professora do Departamento de Saúde Mental e Saúde Coletiva, Universidade Estadual de Montes Claros (Unimontes), Montes Claros, Minas Gerais, Brasil. 3 Doutorando em Saúde Pública, Professor do Departamento de Medicina, Universidade Federal de São João del Rei, Dom Bosco, Minas Gerais. Professor do Departamento de Enfermagem da Universidade Vale do Rio Verde,m Campus Betim, Minas Gerais, Brasil. 4 Doutor em Farmacologia Bioquimica e Molecular, Professor do Departamento de Odontologia, Universidade Estadual de Montes Claros (Unimontes), Montes Claros, Minas Gerais, Brasil. 5 Doutor em Engenharia Elétrica, Professor do Departamento de Ciências da Computação, Universidade Estadual de Montes Claros (Unimontes), Montes Claros, Minas Gerais, Brasil. 6 Doutora em Ciências, Professora do Departamento de Enfermagem, Universidade Estadual de Montes Claros, (Unimontes), Montes Claros, Minas Gerais, Brasil.

\section{RESUMO}

Objetivo: avaliar a qualidade de vida de idosos atendidos em um centro de referência de Minas Gerais. Material e Métodos: pesquisa quantitativa e transversal, realizada em Montes Claros, Minas Gerais - Brasil. Utilizou-se o Instrumento de Avaliação da Qualidade de Vida da Organização Mundial de Saúde para Idosos (WHOQOL-old) para coleta dos dados com uma amostra de 300 idosos. A análise dos dados foi realizada no programa Statistical Package for Social Science, versão 18.0, utilizando das técnicas estatísticas descritivas e clusterização. Resultados: a mensuração da qualidade de vida em todas as dimensões apresentou uma média no escore global de 88,97\%. Observou-se que a dimensões "Morte e Morrer" $(78,13 \%)$ recebeu o maior valor médio. A dimensão "Autonomia" (55,65\%), por sua vez, recebeu o menor valor médio. A clusterização fuzzy apresentou como o maior grau de pertinência a classe "Muito Satisfeito". Conclusão: observou-se que a maioria dos idosos está muito satisfeita com a vida. Ressalta-se a importância em realizar outros estudos com este tipo de corte e população.

Palavras-chave: Serviços de saúde para idosos. Saúde do idoso. Qualidade de vida.

\section{ABSTRACT}

Objective: evaluate the quality of life of elderly in a reference center of Minas Gerais. Methods: quantitative research held in Montes Claros, Minas Gerais - Brazil, using the WHOQOL-old for collection of data on a sample of 300 seniors. Data analysis was carried out in the Statistical Package for Social Science program, version 18.0, using the descriptive statistical techniques, and clustering. Results: the measurement of the quality of life in all dimensions presented an average overall score at 88.97. It was observed that the dimensions "Death and Dying" (78.13\%) received the largest average value. The dimension "autonomy" (55.65\%), in turn, received the lowest average value. Fuzzy clustering presented as the highest degree of relevance the class "Very satisfied". Conclusion: it was noted that most seniors are very satisfied with life. Stresses the importance to undertake other studies with this type of cut and population.

Keywords: Health services for the aged. Health of the elderly. Quality of life.

Autor correspondente: Luís Paulo Souza e Souza, Rua Desembargador Jorge Fontana, 700, apartamento 2002, Belvedere, Belo Horizonte, Minas Gerais. CEP: 30320-670. Telefone: +55 38 99138-1405. E-mail: luis.pauloss12@gmail.com

Conflito de interesses: Não há qualquer conflito de interesses por parte de qualquer um dos autores.

Recebido em: 13 Jan 2017; Revisado em: 24 Fev 2017; Aceito em: 03 Mar 2017. 


\section{INTRODUÇÃO}

As alterações demográficas no mundo têm alertado quanto às peculiaridades envolvidas no processo do envelhecimento. Nos países em desenvolvimento, essas mudanças tornam-se essenciais pela acelerada transição que se associa às dificuldades decorrentes das desigualdades socioeconômicas, que tendem a exigir dos governantes a formulação e adequação de políticas públicas, na busca por um envelhecimento populacional saudável. ${ }^{1}$

A Organização Mundial da Saúde define a população idosa como aquela a partir dos 60 anos de idade, sendo este limite válido para os países em desenvolvimento. Quando se fala em países desenvolvidos, este valor sobe para 65 anos de idade. A estimativa é de que, em 2025, existirão 1,2 bilhões de pessoas com mais de 60 anos no mundo; e que em 2050, haverá cerca de 400 milhões de idosos com mais de 80 anos, frente aos 14 milhões que haviam em meados do século $\mathrm{XX} .^{2}$

Entende-se como envelhecimento um processo do desenvolvimento humano, inerente à vida, com características específicas. O envelhecer provoca no organismo modificações biológicas, psicológicas e sociais que aparecem de forma mais evidente quando alcançada a terceira idade. Assim, o processo de envelhecimento altera a vida dos indivíduos, as estruturas familiares, a demanda por políticas públicas e a distribuição de recursos na sociedade. ${ }^{3}$

A "Qualidade de Vida na velhice" é um conceito relevante na realidade brasileira, pois há uma nova sensibilidade tanto na visão da saúde como na atuação social, sendo considerada como um problema ou desafio para os indivíduos. ${ }^{4}$

O termo "Qualidade de Vida" é definido pela Word Health Organization Quality (WHOQOL-Group) como: "percepção que o indivíduo tem de sua posição na vida dentro do contexto de sua cultura e do sistema de valores de onde vive, e em relação a seus objetivos, expectativas, padrões e preocupações".

A boa qualidade de vida do idoso está relacionada com a capacidade funcional, que é associada à independência e autonomia; e a funcionalidade na velhice, que é influenciada pelo processo de envelhecimento fisiológico por características de gênero, idade, classe social, renda, escolaridade, condições de saúde, cognição, ambiente, história de vida e por recursos de personalidade. Assim, conhecer a qualidade de vida do idoso é relevante para nortear condutas, tratamentos, políticas e proporcionar melhoria na atenção à saúde do idoso. ${ }^{6}$

O objetivo deste estudo foi mensurar e avaliar a qualidade de vida de idosos atendidos em um Centro de Referência no estado de Minas Gerais, região Sudeste do Brasil.

\section{MATERIAL E MÉTODOS}

Trata-se de uma pesquisa de abordagem quantitativa, transversal, realizada no Centro de Referência em Assistência à Saúde do Idoso (CRASI), na cidade de Montes Claros, Estado de Minas Gerais, região Sudeste do Brasil.
O Centro de Referência constitui um ponto de atenção da Rede de Atenção à Saúde do Idoso, recebendo idosos em situação de alto risco ou idosos considerados frágeis, referenciados por alguma equipe da Atenção Primária à Saúde por meio de agendamento pela Central de Regulação Assistencial, e sob supervisão da Secretaria Municipal de Saúde de Montes Claros. A sua área de atuação envolve todo o Norte do Estado de Minas Gerais, sendo o atendimento eletivo e totalmente financiado pelo Sistema Único de Saúde do Brasil (SUS).

O CRASI foi escolhido como cenário deste estudo por ser um serviço inovador com o objetivo de prevenção, promoção e reabilitação de idosos.

Foram incluídos como elegíveis para este estudo todos os idosos cadastrados no centro. $\mathrm{O}$ cálculo amostral foi realizado obtendo como base uma população finita, com a seguinte fórmula: $n$ $=\mathrm{N} \cdot \mathrm{p} 1 \cdot q \cdot \mathrm{z}^{2} /\left[\mathrm{p} 2 \cdot \mathrm{q} \cdot \mathrm{z}^{2}+(\mathrm{N}-1) \cdot \mathrm{e}^{2}\right]$, em que " $\mathrm{n}$ “ é o tamanho da amostra, "n" é o tamanho da população; "p 1" é igual a 0,5 (proporção populacional de indivíduos que pertencem à categoria que estamos interessados em estudar); "p 2" é igual a 0,5 (proporção populacional de indivíduos que não pertencem à categoria que estamos interessados em estudar $(\mathrm{q}=1-\mathrm{p} 2)$ ); "z" é igual a 1,96 (valor crítico que corresponde ao grau de confiança desejado); "e" é igual a 0,5 (5\%) (margem de erro ou erro máximo da estimativa, e identifica a diferença máxima entre a proporção amostral e a verdadeira proporção populacional [p1]). Assim, o cálculo amostral definiu a necessidade de alocação de 300 usuários, considerando-se um erro amostral de $5 \%$ e nível de confiança de $95 \%$, a partir de uma prevalência do evento estudado de 50\% (utilização do serviço de saúde). Esse valor é habitualmente tomado como uma estimativa conservadora, pois aumenta o número da amostra.

Para coleta dos dados, utilizou-se o Instrumento de Avaliação da Qualidade de Vida da Organização Mundial de Saúde para Idosos (WHOQOL-OLD). O WHOQOL-old foi validado no Brasil na versão em português por Fleck, Chachamovick, Trentini. ${ }^{7}$ É constituído por 24 questões fechadas, do tipo escala de likert de cinco pontos e dividido em seis facetas. As facetas são compostas por Funcionamento Sensório (FS); Autonomia (AUT); Atividades passadas, presentes e futuras (PPF); Participação Social (PSO); Morte ou Morrer (MM); Intimidade (INT). ${ }^{7}$

Os dados foram coletados no período de janeiro a fevereiro de 2015, no CRASI, por meio de entrevista direta, devido às dificuldades para leitura apresentado pelos idosos neste Centro de Referência. Os idosos foram abordados, aleatoriamente, no momento de chegada ao Centro, na sala de espera das consultas. Após serem esclarecidos quantos aos objetivos da pesquisa, foram encaminhados a uma sala reservada, com privacidade, para que o instrumento fosse aplicado.

O processamento e a análise dos dados foram realizados no programa Statistical Package for Social Science (SPSS), versão 18.0. Os dados foram analisados através da estatística descritiva (frequência, média, mediana e desvio padrão), por 
meio de cálculos para o Escore Bruto das Facetas (EBF), Escore Médio Padronizado da Faceta (EPF), com valores entre 1 a 5 e o Escore Transformado da Faceta (ETF) que varia de 0 a 100. ${ }^{8-9}$ O escore médio em cada uma das seis facetas indica a percepção dos idosos quanto à sua satisfação em cada um desses aspectos em sua vida, relacionando-os com a sua qualidade de vida. De acordo com a escala de 0 a 100, quanto mais próximo o escore médio dos idosos estiver de 100, mais satisfeita é a percepção acerca daquela faceta. A transformação de um escore bruto para um escore transformado da escala (ETE) entre o 0 e 100 possibilita expressar o escore da escala em percentagem entre o valor mais baixo possível (0) e o mais alto possível (100) de classificação de Qualidade de Vida (QV). ${ }^{8}$

Utilizou-se o corte de clusterização, que é realizado pelo algoritmo fuzzy c-means, que foi um dos primeiros algoritmos propostos para análise de agrupamento (clusterização) fuzzy. Nesse algoritmo, os agrupamentos são representados como um conjunto $C=\left\{\overrightarrow{C_{1}}, \overrightarrow{C_{2}}, \ldots, \overrightarrow{C_{c}}\right\}$ de vetores chamados "protótipos". Cada vetor protótipo sempre está associado à representação de uma classe ou grupo do conjunto de dados e, para isso, deve residir no mesmo espaço menor que os dados do conjunto. ${ }^{9}$

Os aspectos éticos deste estudo foram todos respeitados, baseados na Resolução $n^{\circ}$ 466/12 referente à pesquisa com seres humanos no Brasil. Todos os participantes assinaram o Termo de Consentimento Livre e Esclarecido. O estudo foi aprovado pelo Comitê de Ética em Pesquisa da Universidade Estadual de Minas Gerais, por meio do parecer ${ }^{\circ}$ 890.226.

\section{RESULTADOS}

Quanto à caracterização da amostra, a maioria apresentava 60 a 79 anos de idade; era alfabetizada; do sexo feminino; casada, seguida por viúvas; apresentava renda de um a dois salários mínimos; e possuía casa própria com saneamento básico.

A Tabela 1 mostra o resultado da análise descritiva das dimensões na escala de 0 a 100 da qualidade de vida dos idosos pesquisados.

A mensuração da qualidade de vida em todas as dimensões do WHOQOL-old apresentou uma média no escore global de $88,97 \pm 12,440$. Observou-se que as dimensões "Morte e Morrer" (78,13 $\pm 18,81)$; “Funcionamento Sensório" (74,23 $\pm 27,77)$; "Atividades Passadas, Presentes e Futuras" $(72,46 \pm 17,88)$ receberam os maiores valores médio. As dimensões "Autonomia" $(55,65 \pm 17,97)$; "Participação social" $\quad(59,50 \pm 17,20)$; "Intimidade" $(66,13 \pm 20,03)$ receberam o menor valor médio.

A clusterização fuzzy resultou em uma separação dos dados em três classes: "Muito Satisfeito", "Satisfeito" e "Pouco Satisfeito", apresentando com o maior grau de pertinência na classe "Muito Satisfeito". As medianas dos valores de pertinência de cada classe são ilustradas no Gráfico 1 (boxplot das pertinências encontradas pelo algoritmo fuzzy c-means). A frequência de cada classe no conjunto total dos dados, sendo "1 = Muito Satisfeito", "2 = Pouco Satisfeito" e "3= Satisfeito", é apresentada no Gráfico 2, considerando que a classe escolhida é a que tem o maior grau de pertinência.

Tabela 1. Resultado da análise descritiva das dimensões na escala de 0 a 100 da qualidade de vida dos idosos no Centro de Referência em Assistência à Saúde do Idoso (n=300). Montes Claros, Minas Gerais, Brasil, 2015.

\begin{tabular}{lccccccc}
\hline & $\begin{array}{c}\text { Funcionamento } \\
\text { sensório }\end{array}$ & Autonomia & $\begin{array}{c}\text { Atividades passadas, } \\
\text { presentes e futuras }\end{array}$ & $\begin{array}{c}\text { Participação } \\
\text { social }\end{array}$ & $\begin{array}{c}\text { Morte e } \\
\text { morrer }\end{array}$ & Intimidade & Escore total \\
\hline Média & 74,23 & 55,65 & 72,46 & 59,50 & 78,13 & 66,13 & 88,97 \\
Mediana & 87,50 & 56,25 & 75,00 & 62,50 & 81,25 & 68,75 & 89,00 \\
Desvio Padrão & 27,77 & 17,97 & 17,88 & 17,20 & 18,81 & 20,03 & 12,44 \\
Mínima & 6 & 13 & 13 & 6 & 19 & 13 & 52 \\
Máxima & 100 & 100 & 100 & 100 & 100 & 100 & 117 \\
\hline
\end{tabular}

Gráfico 1. Boxplot das pertinências encontradas pelo algoritmo fuzzy c-Means. Montes Claros, Minas Gerais, Brasil, 2015.

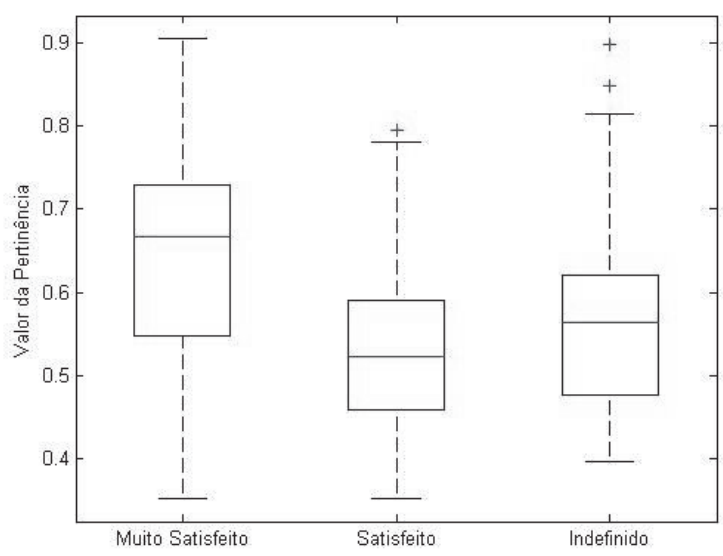

Gráfico 2. Frequência de cada classe no conjunto total dos dados ( $\mathrm{n}=300)$. Montes Claros, Minas Gerais, Brasil, 2015.

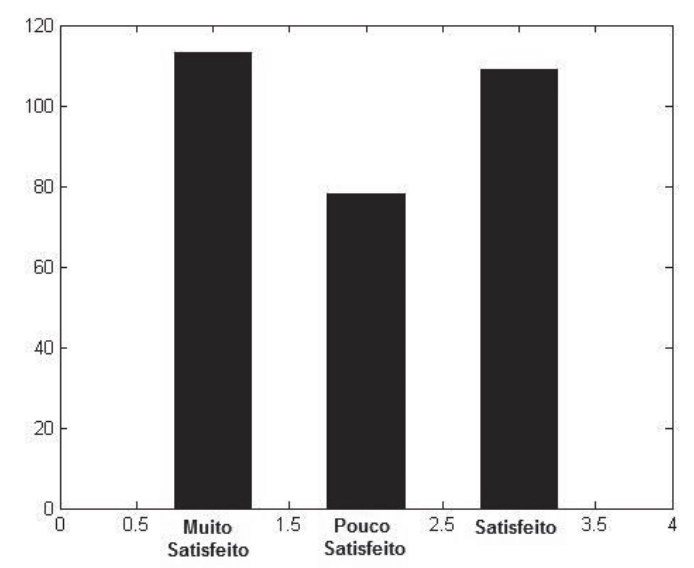




\section{DISCUSSÃO}

Identificou-se que a média global da qualidade de vida dos participantes deste estudo apresentou um valor satisfatório, sendo próximo a $100(88,97)$. Este resultado difere do apresentado por uma pesquisa em São Paulo - Brasil $(67,4)^{4}$ e por outra conduzida em Portugal $(62,88) .{ }^{10}$ Rodrigues e colaboradores ${ }^{11}$ indicam que, por haver divergências destes resultados, há necessidade da realização de estudos mais aprofundados sobre este assunto.

A pontuação advinda do domínio "Morte e Morrer" foi a maior, assim como em outra pesquisa conduzida no Brasil, ${ }^{12}$ ressaltando que o controle de preocupação e temores sobre a morte para os idosos influenciou no escore total de QV, o que pode ser explicado pelo confronto com a morte na medida em que os anos passam e a perda de parentes e amigos é comum. Já em outra investigação no Sul do Brasil, ${ }^{13}$ observou-se um escore inferior $(27,88)$, retratando a pouca preocupação e a coragem dos idosos quando o assunto é a morte.

O "Funcionamento Sensório" foi o segundo maior escore, semelhante aos achados do estudo em Uberaba, no Triângulo de Minas Gerais (média 80,00). ${ }^{14}$ Para os autores, ${ }^{14}$ é importante identificar as dificuldades sensoriais de todos os idosos, com o intuito de diferenciar alterações fisiológicas do processo de envelhecimento e adotar condutas adequadas para manutenção da QV relacionada ao seu funcionamento.

A dimensão "Atividades passadas, presentes e futuras" foi a terceira com maior valor no escore e apresenta resultados controversos a outro estudo. ${ }^{15}$ As conquistas obtidas ao longo da vida e as possibilidades de se conseguirem outras podem estar relacionadas à disposição física do idoso somada às condições socioeconômicas para concretizá-las. ${ }^{16}$

No domínio "Intimidade", evidenciou-se escore razoável $(66,13)$, semelhante ao encontrado no estudo em Portugal $(63,33)^{10}$ e em Arroio do Meio - Brasil $(69,12){ }^{13}$ Estes achados demonstram que, neste domínio, por investigar companheirismo e amor, os idosos apresentavam envelhecimento pleno de satisfação com seus companheiros e com aqueles que os rodeavam.

Como um dos menores escores, a "Participação Social" avaliada neste estudo foi semelhante ao encontrado por outra pesquisa no Brasil, ${ }^{12}$ que encontrou escore de 14,93 para um grupo de idosos e 9,30 para o outro. A participação social pode favorecer as relações sociais, aumentar a rede de apoio e autoestima do idoso, fazendo com que se sinta útil e valorizado na comunidade. Contrapondo, pesquisadores em Foz do Iguaçu - Brasil ${ }^{17}$ encontraram um escore alto para este domínio e consideraram que a participação social reduz com o passar da idade, evidenciado especialmente quando os idosos ultrapassam os 80 anos e quando são mulheres. Para os autores, ${ }^{17}$ isto se deve ao fato de que, nesta etapa, as limitações do processo de envelhecer estão presentes de forma mais aparentes, fazendo com que os idosos permaneçam maior tempo junto à família.
Em relação à dimensão "Autonomia", a avaliação com menor escore encontrada pode ser explicada pelo fato da maioria dos idosos morarem com os filhos, o que pode influenciar na diminuição do poder de decisão em decorrência do possível excesso de cuidado que os familiares tendem a manter sobre o idoso. ${ }^{16}$ Os menores escores de QV entre os idosos evidenciam a necessidade de identificar quais fatores tem contribuído para estes resultados. ${ }^{18}$

$\mathrm{Na}$ análise de cluster, a frequência em cada condição (classe) foi alta para "Muito Satisfeito", intermediária para condição de "Satisfeito" e com menor frequência para a condição "Pouco Satisfeito". Já na análise realizada em Sete Lagoas - Minas Gerais, ${ }^{19}$ foi identificada com destaque a classe "Intermediário", seguida pela classe "Boa" e, com menor valor, a classe "Pior". No estudo em Belo Horizonte - Minas Gerais,${ }^{20}$ realizou-se o mesmo tipo de corte, contudo, foi utilizado o instrumento WHOQOL-bref nos idosos, apresentando resultado alto na classe "Muito Satisfeito", como neste estudo. Assim, observa-se que os idosos apresentavam boa qualidade de vida.

É importante reforçar que, neste estudo, a amostra de idosos analisada foi uma das maiores quando comparada aos estudos aqui citados. Isso pode ser fator potencializador dos resultados apresentados.

\section{CONCLUSÃO}

Observou-se escore alto de qualidade de vida $(88,97)$ entre os idosos. Apesar deste resultado, constatou-se que a morte preocupa esta população, evidenciando a necessidade de criar estratégias para amenizar o sofrimento, como o acompanhamento psicológico. A autonomia foi outro desafio, uma vez que interfere na qualidade de vida e é importante para a manutenção do idoso. Dessa forma, é relevante implantar grupos de apoio aos idosos e aos familiares, com o intuito de elevar a autonomia desta população.

Como limitação deste estudo, destaca-se ter sido aplicado em somente um Centro de Referência, restringindo os achados da população.

Observou-se que a clusterização possibilitou confirmar que a maioria da população estudada está muito satisfeita com a vida. Assim, é válida a realização de outros estudos com este tipo de corte. O WHOQOL-old é um instrumento pertinente para a avaliação da qualidade de vida, pois possibilita detectar as dificuldades dadas no envelhecimento, sendo um importante instrumento para o planejamento de intervenções voltadas à promoção da saúde.

Por fim, reforça-se a necessidade de realização de mais pesquisas com este público, de forma a conhecer como vivem os idosos e propor intervenções que melhorem a qualidade de vida desta população que tem atingindo proporções cada vez maiores no Brasil e no mundo. 


\section{REFERÊNCIAS}

1. Oliveira ER, Gomes MJ, Paiva KM. Institucionalização e qualidade de vida de idosos da região metropolitana de Vitória - ES. Esc Anna Nery. 2011;15(3):518-23.

2. Organização Mundial de Saúde. Envelhecimento ativo: uma política de saúde. Brasília: Organização Pan-Americana da Saúde; 2005.

3. Santos SS. Concepções teórico-filosóficas sobre envelhecimento, velhice, idoso e enfermagem gerontogeriátrica. Rev Bras Enferm. 2010;63(6):1035-9.

4. Gutierrez BA, Auricchio AM, Medina NV. Mensuração da qualidade de vida de idosos em centros de convivência. J Health Sci Inst. 2011;29(3):186-90.

5. WHOQOL Group. The World Health Organization quality of life assessment (WHOQOL): position paper from the World Health Organization. Soc Sci Med. 1995;41(10):1403-9.

6. Stival MM, Lima LR, Funghetto SS, Silva AO, Pinho DL, Karnikowski MG. Fatores associados à qualidade de vida de idosos que frequentam uma unidade de saúde do Distrito Federal. Rev Bras Geriatr Gerontol. 2014;17(2):395-405.

7. Fleck MP, Chachamovich E, Trentini C. Development and validation of the Portuguese version of the WHOQOL-Old module. Rev Saude Publica. 2006;40(5):785-91.

8. Fleck MP, Louzada S, Xavier M, Chachamovich E, Vieira G, Santos L, et al. Aplicação da versão em português do instrumento abreviado de avaliação da qualidade de vida "WHOQOL-Bref". Rev Saude Publica. 2000;34(2):178-83.

9. Nock R, Nielsen F. On weighting clustering. IEEE Trans Pattern Anal Mach Intell. 2006;28(8):1-13.

10. Cimirro PA, Rigon R, Vieira MM, Pereira HM, Creutzberg M. Qualidade de vida de idosos dos centros-dia do Regado e São tomé Portugal. Enfermagem em Foco. 2011;2(3):195-8.

11. Rodrigues SM, Oliveira AC, Vargas AM, Moreira NA, Ferreira EF. Implicações do edentulismo na qualidade de vida entre os idosos. Int J Environ Res Public Health. 2012;9(1):100-9.
12. Maués CR, Paschoal SM, Jaluul O, França CC, Jacob W Filho. Avaliação da qualidade de vida: comparação entre idosos jovens e muito idosos. Rev Bras Clin Med. 2010;8(5):405-10.

13. Bajotto AP, Goldim JR. Avaliação da qualidade de vida e tomada de decisão em idosos participantes de grupos socioterápicos da cidade de Arroio do Meio, RS, Brasil. Rev Bras Geriatr Gerontol. 2011;14(4):753-61.

14. Tavares DM, Araújo MO, Dias FA. Qualidade de vida dos idosos: comparação entre os distritos sanitários de Uberaba-MG. Cienc Cuid Saude. 2011;10(1):74-81.

15. Conrad I, Matschinger H, Riedel-Heller S, Gottberg C, Kilian R. The psychometric properties of the German version of the WHOQOL-OLD in the German population aged 60 and older. Health Qual Life Outcomes. 2014;12:105.

16. Santos EA, Tavares DM, Rodrigues LR, Dias FA, Ferreira PC. Morbidades e qualidade de vida de idosos com diabetes mellitus residentes nas zonas rural e urbana. Rev Esc Enferm USP. 2013;47(2):393-400.

17. Faller JW, Melo WA, Versa GL, Marcon SS. Qualidade de vida de idosos cadastrados na estratégia saúde da família de Foz do IguaçuPR. Esc Anna Nery. 2010;14(4):803-10.

18. Tavares DM, Santos LL, Dias FA, Ferreira PC, Feliciano EA, Mesquita JS. Comparação das características sociodemográficas de saúde e qualidade de vida de idosos rurais segundo sexo. REAS. 2013;2(1):32-46.

19. Campos AC, Ferreira EF, Vargas AM, Albala C. Aging, Gender and Quality of Life (AGEQOL) study: factors associated with good quality of life in older Brazilian community-dwelling adults. Health Qual Life Outcomes. 2014;12:166.

20. Silva PA, Soares SM, Santos JF, Silva LB. Ponto de corte para o WHOQOL-bref como preditor de qualidade de vida de idosos. Rev Saúde Pública. 2014;48(3):390-7.

\section{Como citar:}

Andrade JS, Brito MF, Souza LP, Guimarães AL, D’Angelo MF, Silva CS. Qualidade de vida de idosos atendidos em um centro de referência em Minas Gerais, Brasil. Rev Med UFC. 2018 jan-mar;58(1):26-30. 